Interdisciplinary Studies of Complex Systems

No. 17 (2020) 34-42

(C) Yu. Kondratiev

https://doi.org/10.31392/iscs.2020.17.034

\title{
Philosophy of Natural Numbers
}

\author{
Yuri Kondratiev ${ }^{1,2}$
}

\begin{abstract}
We discuss an extension of classical combinatorics theory to the case of spatially distributed objects.

Keywords: combinatorics, Newton polynomials, Stirling operators, corre-
\end{abstract} lation functions

2010 Mathematics Subject Classification: 60J25, 60J65, 60G22, 47A30

\section{Introduction}

The set of natural numbers $\mathbb{N}=\{0,1,2, \ldots\}$ is a fundamental object in the mathematics. In certain sense $\mathbb{N}$ is the root of all modern mathematics. Other mathematical structures may be created as a logical development of this object. The latter motivated L. Kronecker who summarized "God made the integers, all else is the work of man". There is famous citation from I. Kant: "Two things fill the mind: the starry heavens above me and the moral law within me". A mathematician may continue: "and natural numbers given to my mind".

From the time of Pithagoras philosophers was trying to see hidden meaning of natural numbers and their mystical properties. Considering $\mathbb{N}$ as a set of real things in mathematics we will ask ourself about possible ideas behind these numbers. The myth of Plato's Cave served as one of the motivations for developing his concept of a world of ideas and a world of things. In the dialogue "State" he gives several examples illustrating this concept. As we know, Plato considered mathematics as one of the most important building blocks used to construct his philosophical system. Mathematical theories can serve as simple and illustrative tools for the existence of a world of ideas and a world of things. In a number of model situations, we are dealing with objects that appear from our observations in physics, biology, ecology, etc., yet full understanding of the mathematical structures of these models requires consideration of more general mathematical theories, which under some canonical mapping leads to the model situations in question.

The first and essentially obvious observation here is the following. A number $n \in \mathbb{N}$ we interpret as a number of objects (a population) located in a location space $X$. For simplicity we take $X=\mathbb{R}^{d}$. The collection of all $n$-point subsets (or configurations with $n$ elements) form a locally compact space $\Gamma^{(n)}\left(\mathbb{R}^{d}\right)$.

\footnotetext{
${ }^{1}$ Bielefeld University, Germany. kondrat@math.uni-bielefeld.de

${ }^{2}$ National Pedagogical Dragomanov University, Kyiv, Ukraine
} 
It is the space (quite huge) of ideas for the number $n$. Then to $\mathbb{N}$ corresponds the set

$$
\Gamma_{0}\left(\mathbb{R}^{d}\right)=\cup_{n=0}^{\infty} \Gamma^{(n)}\left(\mathbb{R}^{d}\right)
$$

of all finite configurations. We can consider additionally the set $\Gamma\left(\mathbb{R}^{d}\right)$ consisting all locally finite configurations. This set may be considered as the space of ideas which corresponds to natural numbers and additionally to the actual infinity which is absent in the classical framework on natural numbers.

In such extension of $\mathbb{N}$ we arrive in the main question. Namely, most important mathematical theories related to natural numbers we need to develop to this new level. It concerns, first of all, the combinatorics that play central role in many mathematical structures and applications from probability theory to genetics. In this note we will try to show such possibility trying to be as much as possible on technically simple ground. To be friendly to more wide audience, we restrict out explanations to descriptions of main constructions and formulation of some particular results. For detailed discussions and extended references we refer to the recent paper [2].

\section{Classical combinatorics}

The combinatoric is dealing with the set of natural numbers $\mathbb{N}$ and relations between them. As an important object we introduce binomial coefficients:

$$
\left(\begin{array}{l}
n \\
k
\end{array}\right)=\frac{n(n-1) \ldots(n-k+1)}{k !}
$$

defined for $n \in \mathbb{N}$ and $0 \leq k \leq n$. Introducing the falling factorial $(n)_{k}$ we can write

$$
\left(\begin{array}{l}
n \\
k
\end{array}\right)=\frac{(n)_{k}}{k !}
$$

These coefficients may be extended using embedding $\mathbb{N} \subset \mathbb{R}$ to polynomials

$$
N_{k}(t):=\left(\begin{array}{l}
t \\
k
\end{array}\right)=\frac{t(t-1) \ldots(t-k+1)}{k !}=\frac{(t)_{k}}{k !}, t \in \mathbb{R}
$$

which are called Newton polynomials. For Newton polynomials hold ChuVandermond relations:

$$
(t+s)_{n}=\sum_{k=0}^{n}\left(\begin{array}{l}
n \\
k
\end{array}\right)(t)_{k}(s)_{n-k}
$$

An alternative definition is given by the generation function

$$
e_{\lambda}(t):=e^{t \log (1+\lambda)}=\sum_{n=0}^{\infty} \frac{\lambda^{n}}{n !}(t)_{n}=\sum_{n=)}^{\infty} \lambda^{n} N_{n}(t) .
$$

Such transition to continuous variables makes possible to apply in discrete mathematics methods of analysis. Note that using many particular generation functions we may create different polynomial systems. 
Transition to continuous variables makes possible to apply in discrete mathematics methods of analysis. In particular, let us define for functions $f: \mathbb{R} \rightarrow \mathbb{R}$ difference operators

$$
\begin{aligned}
& \left(D^{+} f\right)(t)=f(t+1)-f(t), \\
& \left(D^{-} f\right)(t)=f(t-1)-f(t) .
\end{aligned}
$$

By a direct computation we obtain

$$
\begin{gathered}
D^{+}(t)_{n}=n(t)_{n-1}, \\
D^{-}(t)_{n}=-n(t-1)_{n-1} .
\end{gathered}
$$

Additionally,

$$
D^{+} e_{\lambda}(t)=\lambda e_{\lambda}(t)
$$

In this way we arrive in the framework of difference calculus closely related with the combinatorics [3]. There are specific questions inside of difference calculus as, e.g., an analysis of Newton series

$$
\sum_{n=0}^{\infty} a_{n} N_{n}(t)
$$

and many others.

For functions $a: \mathbb{N} \rightarrow \mathbb{R}$ we define $b: \mathbb{N} \rightarrow \mathbb{R}$ as

$$
b=K a, \quad b(n)=\sum_{k=0}^{n}\left(\begin{array}{l}
n \\
k
\end{array}\right) a(k) .
$$

This operator $K$ (aka combinatorial transform) is very useful in combinatorics and its inverse gives so-called inclusion-exclusion formula:

$$
a(n)=\sum_{k=0}^{n}\left(\begin{array}{l}
n \\
k
\end{array}\right)(-1)^{n-k} b(k) .
$$

Note that for $a: \mathbb{N} \rightarrow \mathbb{R}, a(j)=0, j \neq k, a(k)=1$

$$
(K a)(n)=\left(\begin{array}{l}
n \\
k
\end{array}\right)=k ! N_{k}(n) .
$$

\section{Spatial combinatorics}

Any $n \in \mathbb{N}$ we interpret as the size of a population. It is convenient in the study of population models. There is a natural generalization leading to spatial ecological models. Now we would like to consider objects located in a given locally compact space $X$. For simplicity we will work with the Euclidean space $\mathbb{R}^{d}$. For the substitution of $\mathbb{N}$ in this situation we can use two possible sets. Denote $\Gamma\left(\mathbb{R}^{d}\right)$ the set of all locally finite configurations (subsets) from $\mathbb{R}^{d}$.

$$
\Gamma\left(\mathbb{R}^{d}\right)=\left\{\gamma \subset \mathbb{R}^{d}|| \gamma \cap K \mid<\infty \text {, any compact } K \subset \mathbb{R}^{d}\right\} .
$$


It is the first version of the space in the spatial (continuous) combinatoric we will use.

Another possibility, is to introduce the set of all finite configurations $\Gamma_{0}\left(\mathbb{R}^{d}\right)$. Then

$$
\Gamma_{0}\left(\mathbb{R}^{d}\right)=\cup_{n=0}^{\infty} \Gamma^{(n)}\left(\mathbb{R}^{d}\right),
$$

where $\Gamma^{(n)}\left(\mathbb{R}^{d}\right)$ denoted the set of all configurations with $n$ elements. We will see that in the continuous combinatoric the spaces $\Gamma\left(\mathbb{R}^{d}\right)$ and $\Gamma_{0}\left(\mathbb{R}^{d}\right)$ will play very different roles. It is a specific moment related with transition to the continuum. In this sense $\mathbb{N}$ is splitting in these two spaces of configurations that makes corresponding combinatorics essentially more reach and sophisticated.

Configuration spaces present beautiful combinations of discrete and continuous properties. In particular, in these spaces we have interesting differential geometry, differential operators and diffusion processes etc., see e.g. [1]. From the other hand side, discreteness of an individual configuration makes possible to introduce proper analog of the difference calculus.

Note from the beginning, that the analog of the extension $\mathbb{N} \subset \mathbb{R}$ now naturally play the pair $\Gamma\left(\mathbb{R}^{d}\right) \subset \mathcal{M}\left(\mathbb{R}^{d}\right)$ where we have in mind an imbedding of configurations in the space of discrete Radon measures on $\mathbb{R}^{d}$ and, as a result, in the space of all Radon mesures on $\mathbb{R}^{d}$ :

$$
\Gamma(X) \ni \gamma \mapsto \gamma(d x)=\sum_{y \in \gamma} \delta_{y}(d x) \in \mathcal{M}\left(\mathbb{R}^{d}\right) .
$$

Therefore, instead of pair

$$
\mathbb{N} \subset \mathbb{R}
$$

we have

$$
\Gamma\left(\mathbb{R}^{d}\right) \subset \mathcal{M}\left(\mathbb{R}^{d}\right) .
$$

As a result, the transition to "continuous" variables in the considered situation leads to functions on $\mathcal{M}\left(\mathbb{R}^{d}\right)$. In spatial combinatorics many objects will be measure-valued.

Now we will introduce an analog of the generation function from classical combinatorics. For a test function from the Schwarz space of test functions $\mathcal{D}\left(\mathbb{R}^{d}\right) 0 \leq \xi \in \mathcal{D}\left(\mathbb{R}^{d}\right)$ consider a function

$$
E_{\xi}(\omega)=e^{<\ln (1+\xi), \omega>} \omega \in \mathcal{D}^{\prime}\left(\mathbb{R}^{d}\right)
$$

that is a function on the space of Schwarz distributions. The power decomposition w.r.t. $\xi$ gives

$$
E_{\xi}(\omega)=\sum_{n=0}^{\infty} \frac{1}{n !}<\xi^{\otimes n},(\omega)_{n}>.
$$

Generalized kernels $(\omega)_{n} \in \mathcal{D}^{\prime}\left(\mathbb{R}^{n d}\right)$ are called infinite dimensional falling factorials on $\mathcal{D}^{\prime}\left(\mathbb{R}^{d}\right)$. Define binomial coefficients (Newton polynomials) on $\mathcal{D}^{\prime}\left(\mathbb{R}^{d}\right)$ as

$$
\left(\begin{array}{l}
\omega \\
n
\end{array}\right)=\frac{(\omega)_{n}}{n !} .
$$


Note that these objects are defined now on the very big space of distributions. In particular cases we shall restrict them on the space of configuration or Radon measures.

In particular, infinite dimensional Chu-Vandermond relations on configurations is

$$
\left(\gamma_{1} \cup \gamma_{2}\right)_{n}=\sum_{k=0}^{n}\left(\begin{array}{l}
n \\
k
\end{array}\right)\left(\gamma_{1}\right)_{k} \otimes\left(\gamma_{2}\right)_{n-k} .
$$

Theorem 1. For $\omega \in \mathcal{M}\left(\mathbb{R}^{d}\right)$

$$
\begin{gathered}
(\omega)_{0}=1 \\
(\omega)_{1}=\omega, \\
(\omega)_{n}\left(x_{1}, \ldots, x_{n}\right)=\omega\left(x_{1}\right)\left(\omega\left(x_{2}\right)-\delta_{x_{1}}\left(x_{2}\right)\right) \ldots\left(\omega\left(x_{n}\right)-\delta_{x_{1}}\left(x_{n}\right)-\ldots-\delta_{x_{n-1}}\left(x_{n}\right)\right) .
\end{gathered}
$$

In the particular case $\omega=\gamma=\left\{x_{i} \mid i \in \mathbb{N}\right\}$

$$
(\gamma)_{n}=n !\left(\begin{array}{l}
\gamma \\
n
\end{array}\right)=\sum_{\left\{i_{1} \ldots, i_{n}\right\} \subset \mathbb{N}} \delta_{x_{1}} \odot \ldots \odot \delta_{x_{n}},
$$

where $\delta_{x_{1}} \odot \ldots \odot \delta_{x_{n}}$ denotes symmetric tensor product.

We have

$$
\Gamma\left(\mathbb{R}^{d}\right) \ni \gamma \mapsto \gamma(d x) \in \mathcal{M}\left(\mathbb{R}^{d}\right) .
$$

Due to our construction

$$
(\gamma)_{n} \in \mathcal{M}\left(\mathbb{R}^{n d}\right)
$$

is a symmetric Radon masure. Therefore, we arrive in measure valued Newton polynomials. The latter is the main consequence of continuous combinatoric transition.

\section{Difference geometry for spatial combinatorics} ent)

For any $x \in \gamma$ define an elementary Markov death operator (death gradi-

$$
D_{x}^{-} F(\gamma)=F(\gamma \backslash x)-F(\gamma)
$$

and the tangent space $T_{\gamma}^{-}(\Gamma)=L^{2}\left(\mathbb{R}^{d}, \gamma\right)$. Then for $\psi \in C_{0}\left(\mathbb{R}^{d}\right)$

$$
D_{\psi}^{-} F(\gamma)=\sum_{x \in \gamma} \psi(x)(F(\gamma \backslash x)-F(\gamma))
$$

is the directional (difference) derivative.

Similarly, we define for $x \in \mathbb{R}^{d}$

$$
D_{x}^{+} F(\gamma)=F(\gamma \cup x)-F(\gamma)
$$

and the tangent space $T_{\gamma}^{-}(\Gamma)=L^{2}\left(\mathbb{R}^{d}, d x\right)$. Then for $\varphi \in C_{0}\left(\mathbb{R}^{d}\right)$

$$
D_{\varphi}^{+} F(\gamma)=\int_{\mathbb{R}^{d}} \varphi(x)(F(\gamma \cup x)-F(\gamma)) d x
$$

is another directional (difference) derivative. 
For $\varphi \in C_{0}\left(\mathbb{R}^{d}\right)$ define a function

$$
E_{\varphi}(\gamma)=\exp (<\gamma, \log (1+\varphi)>), \quad \gamma \in \Gamma
$$

It is the generation function for the system on falling factorials (Newton polynomials) on $\Gamma$ :

$$
E_{\varphi}(\gamma)=\sum_{n=0}^{\infty} \frac{1}{n !}<\varphi^{\otimes n},(\gamma)_{n}>
$$

Then

$$
D_{\psi}^{+} E_{\varphi}(\gamma)=<\varphi \psi>E_{\varphi}(\gamma)
$$

An explicit formula for the falling factorials (as measures on $\left(\mathbb{R}^{d}\right)^{n}$ ) is

$$
(\gamma)_{n}=\sum_{\left\{x_{1}, \ldots, x_{n}\right\} \subset \gamma} \delta_{x_{1}} \odot \delta_{x_{2}} \odot \cdots \odot \delta_{x_{n}}
$$

where $\delta_{x_{1}} \odot \delta_{x_{2}} \odot \cdots \odot \delta_{x_{n}}$ denotes the symmetric tensor product of measures.

The action of difference derivatives on Newton monomials is given by

$$
\begin{gathered}
D_{\psi}^{+}<\varphi^{(n)},(\gamma)_{n}>=n \int_{\mathbb{R}^{d}} \psi(x)<\varphi^{(n)}(x, \cdot),(\gamma)_{n-1}(\cdot)>d x, \\
D_{\psi}^{-}<\varphi^{(n)},(\gamma)_{n}>=-n \sum_{x \in \gamma} \psi(x)<\varphi^{(n)}(x, \cdot),(\gamma \backslash x)_{n-1}(\cdot)>.
\end{gathered}
$$

\section{$5 \quad$ Stirling kernels}

We have polynomial equality

$$
(\gamma)_{n}=\sum_{k=1}^{n} s_{k}^{n} \gamma^{\otimes k}
$$

where

$$
s_{k}^{n}: \mathcal{D}^{\prime}\left(\mathbb{R}^{k d}\right) \rightarrow \mathcal{D}^{\prime}\left(\mathbb{R}^{n d}\right)
$$

is a linear mapping.

On other side

$$
\gamma^{\otimes n}=\sum_{k=0}^{n} S_{k}^{n}(\gamma)_{k}
$$

where

$$
S_{k}^{n}: \mathcal{D}^{\prime}\left(\mathbb{R}^{k d}\right) \rightarrow \mathcal{D}^{\prime}\left(\mathbb{R}^{n d}\right)
$$

is a linear mapping.

Kernels $s_{k}^{n}$ and $S_{k}^{n}$ we will call Stirling kernels of first and second kind respectively. In the classical combinatorics Stirling coefficients play a very important role. 
For $f^{(n)} \in \mathcal{D}\left(\mathbb{R}^{n d}\right)$

$$
\begin{aligned}
<(\gamma)_{n}, f^{(n)}>=\sum_{k=0}^{n} \frac{n !}{k !}<\gamma^{\otimes k}\left(x_{1}, \ldots, x_{k}\right), & \\
& \sum_{i_{1}+\ldots i_{k}=n} \frac{(-1)^{n+k}}{i_{1} \ldots i_{k}} f^{(n)}(\underbrace{x_{1}, \ldots, x_{1}}_{i_{1} \text { times }}, \ldots, \underbrace{x_{k}, \ldots, x_{k}}_{i_{k} \text { times }})>.
\end{aligned}
$$

For the second kind kernels

$$
\begin{aligned}
<\gamma^{\otimes n}, f^{(n)}>=\sum_{k=0}^{n} \frac{1}{k !}<(\gamma)_{k}\left(x_{1}, \ldots, x_{k}\right), & \\
& \sum_{i_{1}+\ldots i_{k}=n}\left(\begin{array}{c}
n \\
i_{1} \ldots i_{k}
\end{array}\right) f^{(n)}(\underbrace{x_{1}, \ldots, x_{1}}_{i_{1} \text { times }}, \ldots, \underbrace{x_{k}, \ldots, x_{k}}_{i_{k} \text { times }})>.
\end{aligned}
$$

\section{Harmonic analysis on $\Gamma\left(\mathbb{R}^{d}\right)$}

Functions $G: \Gamma_{0}\left(\mathbb{R}^{d}\right) \rightarrow \mathbb{R}$ we call quasi-observables. Note that $G$ restricted on $\Gamma^{(n)}\left(\mathbb{R}^{d}\right)$ is given by a symmetric kernel $G^{(n)}\left(x_{1}, \ldots, x_{n}\right)$ and then

$$
G=\left(G^{(n)}\right)_{n=0}^{\infty} .
$$

Functions $F: \Gamma\left(\mathbb{R}^{d}\right) \rightarrow \mathbb{R}$ we call observables. For a quasi-observable $G$ define an operator

$$
(K G)(\gamma)=\sum_{\eta \subset \gamma,|\eta|<\infty} G(\eta), \quad \gamma \in \Gamma\left(\mathbb{R}^{d}\right)
$$

that is an observable. To be well defined we need certain assumptions about $G$ [4].

For $G_{1}, G_{2}: \Gamma_{0}\left(\mathbb{R}^{d}\right) \rightarrow \mathbb{R}$ define

$$
\left(G_{1} \star G_{2}\right)(\eta)=\sum_{\eta_{1} \cup \eta_{2} \cup \eta_{3}=\eta} G_{1}\left(\eta_{1} \cup \eta_{2}\right) G_{2}\left(\eta_{2} \cup \eta_{3}\right) .
$$

Then

$$
K\left(G_{1} \star G_{2}\right)=K G_{1} K G_{2} .
$$

Let $\mu \in \mathcal{M}^{1}\left(\Gamma\left(\mathbb{R}^{d}\right)\right)$.

$$
\begin{gathered}
K: F u n\left(\Gamma_{0}\right) \rightarrow \operatorname{Fun}(\Gamma), \\
K^{*}: \mathcal{M}^{1}(\Gamma) \rightarrow \mathcal{M}\left(\Gamma_{0}\right), \\
K^{*} \mu=\rho, \quad \rho=\left(\rho^{(n)}\right)_{n=0}^{\infty} .
\end{gathered}
$$

The measure $\rho$ is called correlation measure for $\mu$ (Fourier transform of $\mu$ ).

Assume absolute continuity

$$
d \rho^{(n)}\left(x_{1}, \ldots, x_{n}\right)=\frac{1}{n !} k^{(n)}\left(x_{1}, \ldots, x_{n}\right) d x_{1} \ldots x_{n} .
$$

We call $k^{(n)}\left(x_{1}, \ldots, x_{n}\right), n \in \mathbb{N}$ correlation functions of the measure $\mu$. 
Transition from measures to CFs is one of the main technical aspects of the analysis on CS in applications to dynamical problems.

Alternatively define the Bogoliubov functional

$$
B_{\mu}(\phi)=\int_{\Gamma\left(\mathbb{R}^{d}\right)} e^{<\gamma, \log (1+\phi>)} d \mu(\gamma) .
$$

Assuming $B_{\mu}$ is holomorphic in $\phi \in L^{1}\left(\mathbb{R}^{d}\right)$ we obtain

$$
B_{\mu}(\phi)=\sum_{n=0}^{\infty} \frac{1}{n !} \int k^{(n)}\left(x_{1}, \ldots, x_{n}\right) \phi\left(x_{1}\right) \ldots \phi\left(x_{n}\right) d x_{1} \ldots d x_{n} .
$$

\section{From spatial to classical combinatorics}

Having developed combinatorial structures in the continuum, we may consider the inverse direction. Namely, how looks like our infinite-dimensional objects in the one dimensional reduction. Surprisingly, it may give some new structures even in this classical case.

Let $a, b: \mathbb{N} \rightarrow \mathbb{R}$. Define a convolution

$$
(a \star b)(n)=\sum_{j+k+l=n} a(j+k) b(k+l) .
$$

As before

$$
(K a)(n)=\sum_{k+0}^{n}\left(\begin{array}{l}
n \\
k
\end{array}\right) a(k)
$$

Then

$$
K(a \star b)=K a \cdot K b .
$$

Introduce coherent states

$$
\begin{gathered}
e_{\lambda}(\cdot): \mathbb{N} \rightarrow \mathbb{C}, e_{\lambda}(n)=\lambda^{n}, \quad \lambda \in \mathbb{C} . \\
\left(K e_{\lambda}\right)(n)=(1+\lambda)^{n} .
\end{gathered}
$$

The configuration space $\Gamma\left(\mathbb{R}^{d}\right)$ is the space of microscopic states in the classical statistical physics of continuous systems. A measure $\mu \in \mathcal{M}^{1}\left(\Gamma\left(\mathbb{R}^{d}\right)\right)$ is a macroscopic state of a continuous system in the statistical physics. Coming back we can interpret (a bit naively) a measure $\mu \in \mathcal{M}^{1}(\mathbb{N})$ as a state of $0 D$ system.

For example, the Poisson measure for $\sigma>0$ is defined as

$$
\pi_{\sigma}(n)=e^{-\sigma} \frac{\sigma^{n}}{n !}
$$

Several characteristics we can incorporate in such a case from the analysis on $\Gamma\left(\mathbb{R}^{d}\right)$. Introduce the Bogoliubov functional:

$$
\begin{gathered}
B(\lambda)=\int_{\mathbb{R}_{+}}(1+\lambda)^{x} d \mu(x) . \\
(1+\lambda)^{x}=\sum_{n=0}^{\infty} \frac{\lambda^{n}}{n !}(x)_{n} .
\end{gathered}
$$


Theorem 2. Let $\mu \in \mathcal{M}^{1}\left(\mathbb{R}_{+}\right)$. Then $\mu(\mathbb{N})=1$ iff $B(\lambda)$ has a holomorphic extension.

Similarly we can define correlation measures

$$
\begin{gathered}
\int_{\mathbb{N}}(K a)(x) d \mu(x)=\int_{\mathbb{N}} a(x) d \rho_{\mu}(x) . \\
\rho_{\mu}(n)=\frac{1}{n !} \int_{\mathbb{N}}(x)_{n} d \mu(x)=\sum_{m=n}^{\infty}\left(\begin{array}{c}
m \\
n
\end{array}\right) \mu(m) .
\end{gathered}
$$

\section{Acknowledgment}

The financial support by the Ministry for Science and Education of Ukraine through Project 0119U002583 is gratefully acknowledged.

\section{References}

[1] Albeverio, S., Kondratiev, Y.G., Röckner, M. 1998. Analysis and geometry on configuration spaces. J. Funct. Anal. 154, 444-500.

[2] Finkelshtein, D., Kondratiev, Y., Lytvynov, E., Oliveira, M. J. 2020. Spatial combinatorics. ArXiv 2007.01175v1.

[3] Flajolet, P., Sedgewick, R. 2009. Analytic Combinatorics. Cambridge University Press.

[4] Kondratiev, Y.G., Kuna, T. 2002. Harmonic analysis on configuration space. I. General theory. Infin. Dimens. Anal. Quantum Probab. Relat. Top. 5, 201-233. 